\title{
An Estimate of Lambda in Resummed Quantum Gravity in the Context of Asymptotic Safety and Planck Scale Cosmology: Constraints on SUSY GUTS
}

\section{B.F.L. Ward*}

Baylor University

E-mail: bfl_wardebaylor.edu

\begin{abstract}
We use the amplitude-based resummation of Feynman 's formulation of Einstein 's theory to arrive at a UV finite approach to quantum gravity. We show that we recover the UV fixed point recently claimed by the exact field-space renormalization group approach. We use our approach in the context of the attendant Planck scale cosmology formulation of Bonanno and Reuter to estimate the value of the cosmological constant as $\rho_{\Lambda}=(0.0024 \mathrm{eV})^{4}$. We show that the closeness of this estimate to experiment constrains susy GUT models.
\end{abstract}

36th International Conference on High Energy Physics

4-11 July 2012

Melbourne, Australia

\footnotetext{
* Speaker.
} 


\section{Introduction}

In Ref. [1], Weinberg suggested that the general theory of relativity may be asymptotically safe, with an S-matrix that depends on only a finite number of observable parameters, due to the presence of a non-trivial UV fixed point, with a finite dimensional critical surface in the UV limit. Strong evidence has been calculated [2-7] using Wilsonian [8] field-space exact renormalization group methods to support Weinberg's asymptotic safety hypothesis for the Einstein-Hilbert theory. A parallel but independent development [9-11], has shown [12] that the extension of the amplitude-based, exact resummation theory of Ref. [13] to the Einstein-Hilbert theory leads to UV-fixed-point behavior for the dimensionless gravitational and cosmological constants and to a resummed theory, resummed quantum gravity, that is actually UV finite. More evidence for Weinberg's asymptotic safety behavior has been calculated using causal dynamical triangulated lattice methods in Ref. [14] $]^{1}$.

The results in Refs. [2-7], while impressive, involve cut-offs and some dependence on gauge parameters which remain in the results to varying degrees even for products such as that for the UV limits of the dimensionless gravitational and cosmological constants. Accordingly, we refer to the approach in Refs. [2-7] as the 'phenomenological' asymptotic safety approach. The above noted dependencies are mild enough that the non-Gaussian UV fixed point found in these references is probably a physical result. But, the results cannot be considered final until a rigorously cut-off independent and gauge invariant calculation corroborates them. Our approach offers such a possibility, as our results are both gauge invariant and cut-off independent. The results from Refs. [14] involve lattice constant-type artifact issues - to be considered final they too need to be corroborated by a rigorous calculation without such issues. Again, our approach offers a possible answer. The stage is therefore prepared for us to try to make contact with experiment.

Accordingly, we note that, in Refs. [16,17], it has been argued that the attendant phenomenological asymptotic safety approach in Refs. [2-7] to quantum gravity may indeed provide a realization $^{2}$ of the successful inflationary model $[19,20]$ of cosmology without the need of the inflaton scalar field: the attendant UV fixed point solution allows one to develop Planck scale cosmology that joins smoothly onto the standard Friedmann-Walker-Robertson classical descriptions. One arrives at a quantum mechanical solution to the horizon, flatness, entropy and scale free spectrum problems. In Ref. [12], we have shown that, in the new resummed theory [9-11] of quantum gravity, we reproduce the properties as used in Refs. [16,17] for the UV fixed point of quantum gravity with "first principles" predictions for the fixed point values of the respective dimensionless gravitational and cosmological constants. Here, we carry the analysis forward to arrive at an estimate for the observed cosmological constant $\Lambda$ in the context of the Planck scale cosmology of Refs. $[16,17]$. We comment on the reliability of the result, as it will be seen already to be relatively close to the observed value [21,22]. While we obviously do not want to overdo the closeness to the experimental value, we feel that this again gives, at the least, some more credibility to the new resummed theory as well as to the methods in Refs. $[2-7,14]$. We also show how the closeness of our estimate to the observed value would constrain SUSY GUT models when this closeness is put on a more firm basis. More reflections on such matters will be taken up elsewhere [23].

The discussion is organized as follows. We start by recapitulating in the next section the Planck scale cosmology presented phenomenologically in Refs. [16,17]. We then review briefly in Section 3 our results in Ref. [12] for the dimensionless gravitational and cosmological constants at the UV fixed point. In Section 4, we combine the Planck scale cosmology scenario in Refs. $[16,17]$ with our results to estimate the observed value of the cosmological constant $\Lambda$ and we use it to constrain SUSY GUTs.

\section{Planck Scale Cosmology}

We recall the Einstein-Hilbert theory

$$
\mathscr{L}(x)=\frac{1}{2 \kappa^{2}} \sqrt{-g}(R-2 \Lambda)
$$

where $R$ is the curvature scalar, $g$ is the determinant of the metric of space-time $g_{\mu v}, \Lambda$ is the cosmological constant and $\kappa=\sqrt{8 \pi G_{N}}$ for Newton's constant $G_{N}$. The authors in Ref. [16,17], using

\footnotetext{
${ }^{1}$ We also note that the model in Ref. [15] realizes many aspects of the effective field theory implied by the anomalous dimension of 2 at the UV-fixed point but it does so at the expense of violating Lorentz invariance.

${ }^{2}$ The attendant choice of the scale $k \sim 1 / t$ used in Refs. [16,17] was also proposed in Ref. [18].
} 
the phenomenological exact renormalization group for the Wilsonian [8] coarse grained effective average action in field space, have argued that the attendant running Newton constant $G_{N}(k)$ and running cosmological constant $\Lambda(k)$ approach UV fixed points as $k$ goes to infinity in the deep Euclidean regime in the sense that $k^{2} G_{N}(k) \rightarrow g_{*}, \Lambda(k) \rightarrow \lambda_{*} k^{2}$ for $k \rightarrow \infty$ in the Euclidean regime.

The contact with cosmology proceeds as follows. Using a phenomenological connection between the momentum scale $k$ characterizing the coarseness of the Wilsonian graininess of the average effective action and the cosmological time $t$, the authors in Refs. [16, 17] show that the standard cosmological equations admit of the following extension: $\left(\frac{\dot{a}}{a}\right)^{2}+\frac{K}{a^{2}}=\frac{1}{3} \Lambda+\frac{8 \pi}{3} G_{N} \rho, \dot{\rho}+$ $3(1+\omega) \frac{\dot{a}}{a} \rho=0, \dot{\Lambda}+8 \pi \rho \dot{G}_{N}=0, G_{N}(t)=G_{N}(k(t)), \Lambda(t)=\Lambda(k(t))$ in a standard notation for the density $\rho$ and scale factor $a(t)$ with the Robertson-Walker metric representation as

$$
d s^{2}=d t^{2}-a(t)^{2}\left(\frac{d r^{2}}{1-K r^{2}}+r^{2}\left(d \theta^{2}+\sin ^{2} \theta d \phi^{2}\right)\right)
$$

so that $K=0,1,-1$ correspond respectively to flat, spherical and pseudo-spherical 3-spaces for constant time t. The equation of state is taken as $p(t)=\omega \rho(t)$, where $p$ is the pressure. The attendant functional relationship between the respective momentum scale $k$ and the cosmological time $t$ is determined phenomenologically via $k(t)=\frac{\xi}{t}$ for some positive constant $\xi$ determined from constraints on physically observable predictions.

Using the UV fixed points as discussed above for $k^{2} G_{N}(k) \equiv g_{*}$ and $\Lambda(k) / k^{2} \equiv \lambda_{*}$ obtained from their phenomenological, exact renormalization group (asymptotic safety) analysis, the authors in Refs. $[16,17]$ show that the system given above admits, for $K=0$, a solution in the Planck regime where $0 \leq t \leq t_{\text {class }}$, with $t_{\text {class }}$ a "few" times the Planck time $t_{P l}$, which joins smoothly onto a solution in the classical regime, $t>t_{\text {class }}$, which coincides with standard Friedmann-RobertsonWalker phenomenology but with the horizon, flatness, scale free Harrison-Zeldovich spectrum, and entropy problems all solved purely by Planck scale quantum physics.

While the dependencies of the fixed-point results $g_{*}, \lambda_{*}$ on the cut-offs used in the Wilsonian coarse-graining procedure, for example, make the phenomenological nature of the analyses in Refs. [16,17] manifest, we note that the key properties of $g_{*}, \lambda_{*}$ used for these analyses are that the two UV limits are both positive and that the product $g_{*} \lambda_{*}$ is only mildly cut-off/threshold function dependent. Here, we review the predictions in Refs. [12] for these UV limits as implied by resummed quantum gravity(RQG) theory as presented in [9-11] and show how to use them to predict the current value of $\Lambda$. For completeness, we start the next section with a brief review of the basic principles of RQG theory.

\section{3. $g_{*}$ and $\lambda_{*}$ in Resummed Quantum Gravity}

We start with the prediction for $g_{*}$, which we already presented in Refs. [9-12]. Given that the theory we use is not very familiar, we recapitulate the main steps in the calculation.

As the graviton couples to an elementary particle in the infrared regime which we shall resum independently of the particle's spin [24], we may use a scalar field to develop the required calculational framework, which we then extend to spinning particles straightforwardly. We follow Feynman in Refs. $[25,26]$ and start with the Lagrangian density for the basic scalar-graviton system:

$$
\begin{aligned}
& \mathscr{L}(x)=-\frac{1}{2 \kappa^{2}} R \sqrt{-g}+\frac{1}{2}\left(g^{\mu \nu} \partial_{\mu} \varphi \partial_{\nu} \varphi-m_{o}^{2} \varphi^{2}\right) \sqrt{-g} \\
& =\frac{1}{2}\left\{h^{\mu v, \lambda} \bar{h}_{\mu v, \lambda}-2 \eta^{\mu \mu^{\prime}} \eta^{\lambda \lambda^{\prime}} \bar{h}_{\mu_{\lambda}, \lambda^{\prime}} \eta^{\sigma \sigma^{\prime}} \bar{h}_{\mu^{\prime} \sigma, \sigma^{\prime}}\right\} \\
& +\frac{1}{2}\left\{\varphi_{, \mu} \varphi^{, \mu}-m_{o}^{2} \varphi^{2}\right\}-\kappa h^{\mu v}\left[\overline{\varphi_{, \mu} \varphi_{, v}}+\frac{1}{2} m_{o}^{2} \varphi^{2} \eta_{\mu v}\right] \\
& -\kappa^{2}\left[\frac{1}{2} h_{\lambda \rho} \bar{h}^{\rho \lambda}\left(\varphi_{, \mu} \varphi^{, \mu}-m_{o}^{2} \varphi^{2}\right)-2 \eta_{\rho \rho^{\prime}} h^{\mu \rho} \bar{h}^{\rho^{\prime} v} \varphi_{, \mu} \varphi_{, v}\right]+\cdots
\end{aligned}
$$

Here, $\varphi(x)$ can be identified as the physical Higgs field as our representative scalar field for matter, $\varphi(x)_{, \mu} \equiv \partial_{\mu} \varphi(x)$, and $g_{\mu v}(x)=\eta_{\mu v}+2 \kappa h_{\mu v}(x)$ where we follow Feynman and expand about 


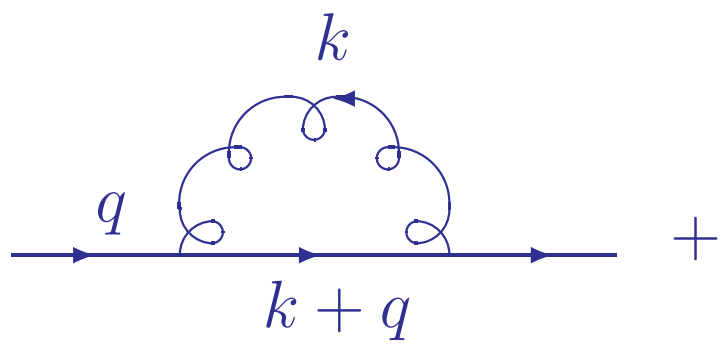

(a)

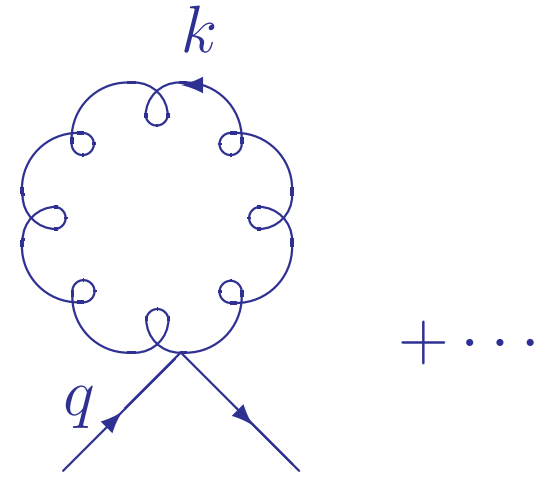

(b)

Figure 1: Graviton loop contributions to the scalar propagator. $q$ is the 4-momentum of the scalar.

Minkowski space so that $\eta_{\mu v}=\operatorname{diag}\{1,-1,-1,-1\}$. We have introduced Feynman's notation $\bar{y}_{\mu \nu} \equiv \frac{1}{2}\left(y_{\mu \nu}+y_{\nu \mu}-\eta_{\mu v} y_{\rho}{ }^{\rho}\right)$ for any tensor $y_{\mu \nu}{ }^{3}$. The bare(renormalized) scalar boson mass here is $m_{o}(m)$ and we set presently the small observed [21,22] value of the cosmological constant to zero so that our quantum graviton, $h_{\mu \nu}$, has zero rest mass. We return to the latter point, however, when we discuss phenomenology. Feynman $[25,26]$ has essentially worked out the Feynman rules for (3.1), including the rule for the famous Feynman-Faddeev-Popov [25,27,28] ghost contribution required for unitarity with the fixing of the gauge (we use the gauge of Feynman in Ref. [25], $\left.\partial^{\mu} \bar{h}_{v \mu}=0\right)$. For this material we refer to Refs. $[25,26]$. We turn now directly to the quantum loop corrections in the theory in (3.1).

Referring to Fig. 1, we have shown in Refs. [9-11] that the large virtual IR effects in the respective loop integrals for the scalar propagator in quantum general relativity can be resummed to the exact result $i \Delta_{F}^{\prime}(k)=\frac{i}{k^{2}-m^{2}-\Sigma_{s}(k)+i \varepsilon}=\left.\frac{i e_{g}^{B_{g}^{\prime \prime}(k)}}{k^{2}-m^{2}-\Sigma_{s}^{\prime}+i \varepsilon} \equiv i \Delta_{F}^{\prime}(k)\right|_{\text {resummed }}$ for $\quad\left(\Delta=k^{2}-m^{2}\right)$

$$
\begin{aligned}
B_{g}^{\prime \prime}(k)= & -2 i \kappa^{2} k^{4} \frac{\int d^{4} \ell}{16 \pi^{4}} \frac{1}{\ell^{2}-\lambda^{2}+i \varepsilon} \\
& \frac{1}{\left(\ell^{2}+2 \ell k+\Delta+i \varepsilon\right)^{2}} \\
= & \frac{\kappa^{2}\left|k^{2}\right|}{8 \pi^{2}} \ln \left(\frac{m^{2}}{m^{2}+\left|k^{2}\right|}\right),
\end{aligned}
$$

where the latter form holds for the UV(deep Euclidean) regime, so that $\left.\Delta_{F}^{\prime}(k)\right|_{\text {resummed }}$ falls faster than any power of $\left|k^{2}\right|$ - by Wick rotation, the identification $-\left|k^{2}\right| \equiv k^{2}$ in the deep Euclidean regime gives immediate analytic continuation to the result in the last line of (3.2) when the usual $-i \varepsilon, \varepsilon \downarrow 0$, is appended to $m^{2}$. An analogous result [9] holds for $\mathrm{m}=0$. Here, $-i \Sigma_{s}(k)$ is the 1PI scalar self-energy function so that $i \Delta_{F}^{\prime}(k)$ is the exact scalar propagator. As $\Sigma_{s}^{\prime}$ starts in $\mathscr{O}\left(\kappa^{2}\right)$, we may drop it in calculating one-loop effects. When the respective analogs of $\left.i \Delta_{F}^{\prime}(k)\right|_{\text {resummed }}{ }^{4}$ are used for the elementary particles, one-loop corrections are finite. In fact, the use of our resummed propagators renders all quantum gravity loops UV finite [9-11]. It is this attendant representation of the quantum theory of general relativity that we have called resummed quantum gravity (RQG).

\footnotetext{
${ }^{3}$ Our conventions for raising and lowering indices in the second line of (3.1) are the same as those in Ref. [26].

${ }^{4}$ These follow from the observation $[9,24]$ that the IR limit of the coupling of the graviton to a particle is independent of its spin.
} 
Indeed, when we use our resummed propagator results, as extended to all the particles in the SM Lagrangian and to the graviton itself, working now with the complete theory $\mathscr{L}(x)=$ $\frac{1}{2 \kappa^{2}} \sqrt{-g}(R-2 \Lambda)+\sqrt{-g} L_{S M}^{\mathscr{G}}(x)$ where $L_{S M}^{\mathscr{G}}(x)$ is SM Lagrangian written in diffeomorphism invariant form as explained in Refs. $[9,11]$, we show in the Refs. [9-11] that the denominator for the propagation of transverse-traceless modes of the graviton becomes $\left(M_{P l}\right.$ is the Planck mass $) q^{2}+$ $\Sigma^{T}\left(q^{2}\right)+i \varepsilon \cong q^{2}-q^{4} \frac{c_{2, e f f}}{360 \pi M_{P l}^{2}}$, where we have defined $c_{2, \text { eff }}=\sum_{\text {SM particles j }} n_{j} I_{2}\left(\lambda_{c}(j)\right) \cong 2.56 \times 10^{4}$ with $I_{2}$ defined [9-11] by $I_{2}\left(\lambda_{c}\right)=\int_{0}^{\infty} d x x^{3}(1+x)^{-4-\lambda_{c} x}$ and with $\lambda_{c}(j)=\frac{2 m_{j}^{2}}{\pi M_{P l}^{2}}$ and [9-11] $n_{j}$ equal to the number of effective degrees of particle $j$. The details of the derivation of the numerical value of $c_{2, \text { eff }}$ are given in Refs. [9]. These results allow us to identify (we use $G_{N}$ for $G_{N}(0)$ ) $G_{N}(k)=$ $G_{N} /\left(1+\frac{c_{2, e f f} k^{2}}{360 \pi M_{P l}^{2}}\right)$ and to compute the UV limit $g_{*}$ as $g_{*}=\lim _{k^{2} \rightarrow \infty} k^{2} G_{N}\left(k^{2}\right)=\frac{360 \pi}{c_{2, e f f}} \cong 0.0442$.

For the prediction for $\lambda_{*}$, we use the Euler-Lagrange equations to get Einstein's equation as

$$
G_{\mu v}+\Lambda g_{\mu \nu}=-\kappa^{2} T_{\mu \nu}
$$

in a standard notation where $G_{\mu \nu}=R_{\mu \nu}-\frac{1}{2} R g_{\mu \nu}, R_{\mu \nu}$ is the contracted Riemann tensor, and $T_{\mu \nu}$ is the energy-momentum tensor. Working then with the representation $g_{\mu \nu}=\eta_{\mu \nu}+2 \kappa h_{\mu \nu}$ for the flat Minkowski metric $\eta_{\mu \nu}=\operatorname{diag}(1,-1,-1,-1)$ we see that to isolate $\Lambda$ in Einstein's equation (3.3) we may evaluate its VEV(vacuum expectation value of both sides). On doing this as described in Refs. [9], we see that a scalar makes the contribution to $\Lambda$ given by ${ }^{5}$

$$
\Lambda_{s}=-8 \pi G_{N} \frac{\int d^{4} k}{2(2 \pi)^{4}} \frac{\left(2 k_{0}^{2}\right) e^{-\lambda_{c}\left(k^{2} /\left(2 m^{2}\right)\right) \ln \left(k^{2} / m^{2}+1\right)}}{k^{2}+m^{2}} \cong-8 \pi G_{N}\left[\frac{1}{G_{N}^{2} 64 \rho^{2}}\right]
$$

where $\rho=\ln \frac{2}{\lambda_{c}}$ and we have used the calculus of Refs. [9-11]. The standard methods [9] then show that a Dirac fermion contributes -4 times $\Lambda_{s}$ to $\Lambda$, so that the deep UV limit of $\Lambda$ then becomes, allowing $G_{N}(k)$ to run, $\Lambda(k) \longrightarrow k^{2} \rightarrow \infty k^{2} \lambda_{*}, \lambda_{*}=-\frac{c_{2, e f f}}{2880} \sum_{j}(-1)^{F_{j}} n_{j} / \rho_{j}^{2} \cong 0.0817$ where $F_{j}$ is the fermion number of $j, n_{j}$ is the effective number of degrees of freedom of $j$ and $\rho_{j}=\rho\left(\lambda_{c}\left(m_{j}\right)\right)$. We note that $\lambda_{*}$ would vanish in an exactly supersymmetric theory.

For reference, the UV fixed-point calculated here, $\left(g_{*}, \lambda_{*}\right) \cong(0.0442,0.0817)$, can be compared with the estimates $\left(g_{*}, \lambda_{*}\right) \approx(0.27,0.36)$ in Refs. [16, 17]. In making this comparison, one must keep in mind that the analysis in Refs. $[16,17]$ did not include the specific SM matter action and that there is definitely cut-off function sensitivity to the results in the latter analyses. What is important is that the qualitative results that $g_{*}$ and $\lambda_{*}$ are both positive and are less than 1 in size are true of our results as well. See Refs. [9] for further discussion of the relationship between our $\left\{g_{*}, \lambda_{*}\right\}$ predictions and those in Refs. [16,17].

\section{An Estimate of $\Lambda$ and Constraints on SUSY GUTS}

The results here, taken together with those in Refs. [16,17], allow us to estimate the value of $\Lambda$ today. We take the normal-ordered form of Einstein's equation

$$
: G_{\mu v}:+\Lambda: g_{\mu v}:=-\kappa^{2}: T_{\mu v}:
$$

The coherent state representation of the thermal density matrix then gives the Einstein equation in the form of thermally averaged quantities with $\Lambda$ given by our result in (3.4) summed over the degrees of freedom as specified above in lowest order. In Ref. [17], it is argued that the Planck scale cosmology description of inflation gives the transition time between the Planck regime and the classical Friedmann-Robertson-Walker(FRW) regime as $t_{t r} \sim 25 t_{P l}$. (We discuss in

\footnotetext{
${ }^{5}$ We note the use here in the integrand of $2 k_{0}^{2}$ rather than the $2\left(\vec{k}^{2}+m^{2}\right)$ in Ref. [12], to be consistent with $\omega=$ -1 [29] for the vacuum stress-energy tensor.
} 
Refs. [9] on the uncertainty of this choice of $t_{t r}$.) We thus start with the quantity $\rho_{\Lambda}\left(t_{t r}\right) \equiv \frac{\Lambda\left(t_{t r}\right)}{8 \pi G_{N}\left(t_{t r}\right)}=$ $\frac{-M_{P l}^{4}\left(k_{t r}\right)}{64} \sum_{j} \frac{(-1)^{F} n_{j}}{\rho_{j}^{2}}$ and employ the arguments in Refs. [30] ( $t_{e q}$ is the time of matter-radiation equality) to get the first principles field theoretic estimate

$$
\begin{aligned}
& \rho_{\Lambda}\left(t_{0}\right) \cong \frac{-M_{P l}^{4}\left(1+c_{2, e f f} k_{t r}^{2} /\left(360 \pi M_{P l}^{2}\right)\right)^{2}}{64} \sum_{j} \frac{(-1)^{F} n_{j}}{\rho_{j}^{2}} \\
& \times \frac{t_{t r}^{2}}{t_{e q}^{2}} \times\left(\frac{t_{e q}^{2 / 3}}{t_{0}^{2 / 3}}\right)^{3} \\
& \cong \frac{-M_{P l}^{2}(1.0362)^{2}\left(-9.194 \times 10^{-3}\right)}{64} \frac{(25)^{2}}{t_{0}^{2}} \\
& \cong\left(2.4 \times 10^{-3} \mathrm{eV}\right)^{4} .
\end{aligned}
$$

where we take the age of the universe to be $t_{0} \cong 13.7 \times 10^{9}$ yrs. In the latter estimate, the first factor in the second line comes from the period from $t_{t r}$ to $t_{e q}$ which is radiation dominated and the second factor comes from the period from $t_{e q}$ to $t_{0}$ which is matter dominated ${ }^{6}$. This estimate should be compared with the experimental result $\left.[22]^{7} \rho_{\Lambda}\left(t_{0}\right)\right|_{\text {expt }} \cong\left((2.37 \pm 0.05) \times 10^{-3} \mathrm{eV}\right)^{4}$.

To sum up, we believe our estimate of $\rho_{\Lambda}\left(t_{0}\right)$ represents some amount of progress in the long effort to understand its observed value in quantum field theory. Evidently, the estimate is not a precision prediction, as hitherto unseen degrees of freedom, such as a high scale GUT theory, may exist that have not been included in the calculation.

We now comment on what would happen to our estimate if there would be a GUT theory at high scale. As is well-known, the main viable approaches involve susy GUT's and for definiteness, we will use the susy SO(10) GUT model in Ref. [32] to illustrate how such theory might affect our estimate of $\Lambda$. In this model, the break-down of the GUT gauge symmetry to the low energy gauge symmetry occurs with an intermediate stage with gauge group $S U_{2 L} \times S U_{2 R} \times U_{1} \times S U(3)^{c}$ where the final break-down to the Standard Model $[33,34]$ gauge group, $S U_{2 L} \times U_{1} \times S U(3)^{c}$, occurs at a scale $M_{R} \gtrsim 2 \mathrm{TeV}$ while the breakdown of global susy occurs at the (EW) scale $M_{S}$ which satisfies $M_{R}>M_{S}$. For our purposes the key observation is that susy multiplets do not contribute to our formula for $\rho_{\Lambda}\left(t_{t r}\right)$ when susy is not broken - there is exact cancellation between fermions and bosons in a given degenerate susy multiplet. Thus only the the broken susy multiplets can contribute. In the model at hand, these are just the multiplets associated with the known SM particles and the extra Higgs multiplet required by susy in the MSSM [35]. In view of recent LHC results [36], we take for illustration the values $M_{R} \cong 4 M_{S} \sim 2$.0TeV and set the following susy partner values: $m_{\tilde{g}} \cong$ $1.5(10) \mathrm{TeV}, m_{\tilde{G}} \cong 1.5 \mathrm{TeV}, m_{\tilde{q}} \cong 1.0 \mathrm{TeV}, m_{\tilde{\ell}} \cong 0.5 \mathrm{TeV}, m_{\tilde{\chi}_{i}^{0}} \cong\left\{\begin{array}{l}0.4 \mathrm{TeV}, i=1 \\ 0.5 \mathrm{TeV}, i=2,3,4\end{array}, m_{\tilde{\chi}_{i}^{ \pm}} \cong\right.$ $0.5 \mathrm{TeV}, i=1,2, m_{S}=.5 \mathrm{TeV}, S=A^{0}, H^{ \pm}, H_{2}$, where we use a standard notation for the susy partners of the known quarks $(q \leftrightarrow \tilde{q})$, leptons $(\ell \leftrightarrow \tilde{\ell})$ and gluons $(G \leftrightarrow \tilde{G})$, and the EW gauge and Higgs bosons $\left(\gamma, Z^{0}, W^{ \pm}, H, A^{0}, H^{ \pm}, H_{2} \leftrightarrow \tilde{\chi}\right)$ with the extra Higgs particles denoted as usual [35] by $A^{0}$ (pseudo-scalar), $H^{ \pm}$(charged) and $H_{2}$ (heavy scalar). $\tilde{g}$ is the gravitino, for which we show two examples of its mass for illustration. These particles then generate the extra contribution $\Delta W_{\rho, \mathrm{GUT}}=\sum_{j \in\{\text { MSSM low energy susy partners }\}} \frac{(-1)^{F} n_{j}}{\rho_{j}^{2}} \cong 1.13(1.12) \times 10^{-2}$ to the factor $W_{\rho} \equiv \sum_{j} \frac{(-1)^{F} n_{j}}{\rho_{j}^{2}}$ on the RHS of our equation for $\rho_{\Lambda}\left(t_{t r}\right)$ for the two respective values of $m_{\tilde{g}}$ called out by the parentheses. The corresponding values of $\rho_{\Lambda}$ are $-\left(1.67 \times 10^{-3} \mathrm{eV}\right)^{4}\left(-\left(1.65 \times 10^{-3} \mathrm{eV}\right)^{4}\right)$, respectively. The sign of these results would appear to put them in conflict with the positive observed value

\footnotetext{
${ }^{6}$ The method of the operator field forces the vacuum energies to follow the same scaling as the non-vacuum excitations.

${ }^{7}$ See also Ref. [31] for an analysis that suggests a value for $\rho_{\Lambda}\left(t_{0}\right)$ that is qualitatively similar to this experimental result.
} 
quoted above by many standard deviations, even when we allow for the considerable uncertainty in the various other factors multiplying $W_{\rho}$ in our formula for $\rho_{\Lambda}\left(t_{t r}\right)$, all of which are positive in our framework. This may be alleviated either by adding new particles to the model, approach (A), or by allowing a soft susy breaking mass term for the gravitino that resides near the GUT scale $M_{G U T}$, which is $\sim 4 \times 10^{16} \mathrm{GeV}$ here [32], approach (B). In approach (A), we double the number of quarks and leptons, but we invert the mass hierarchy between susy partners, so that the new squarks and sleptons are lighter than the new quarks and leptons. This can work as long as as we increase $M_{R}, M_{S}$ so that we have the new quarks and leptons at $M_{\mathrm{High}} \sim 3.4(3.3) \times 10^{3} \mathrm{TeV}$ while leaving their partners at $M_{\text {Low }} \sim .5 \mathrm{TeV}$. For approach (B), the mass of the gravitino soft breaking term should be set to $m_{\tilde{g}} \sim 2.3 \times 10^{15} \mathrm{GeV}$. More generally, our estimate in (4.2) can be used as a constraint of general susy GUT models and we hope to explore such in more detail elsewhere.

Realistically, as we explain in Refs. [9], we stress that we actually do not know the precise value of $t_{t r}$ at this point to better than a couple of orders of magnitude which translate to an uncertainty at the level of $10^{4}$ on our estimate of $\rho_{\Lambda}$. We caution the reader to keep this in mind.

There is one further important matter that we have not mentioned: the effect of the various spontaneous symmetry vacuum energies on our $\rho_{\Lambda}$ estimate. From the standard methods we know for example that the energy of the broken vacuum for the EW case contributes an amount of order $M_{W}^{4}$ to $\rho_{\Lambda}$. If we consider the GUT symmetry breaking we expect an analogous contribution from spontaneous symmetry breaking of order $M_{G U T}^{4}$. When compared to the RHS of our equation for $\rho_{\Lambda}\left(t_{t r}\right)$, which is $\sim\left(-(1.0362)^{2} W_{\rho} / 64\right) M_{P l}^{4} \simeq \frac{10^{-2}}{64} M_{P l}^{4}$, we see that adding these effects thereto would make relative changes in our results at the level of $\frac{64}{10^{-2}} \frac{M_{W}^{4}}{M_{P l}^{4}} \cong 1 \times 10^{-65}$ and $\frac{64}{10^{-2}} \frac{M_{G U T}^{4}}{M_{P l}^{4}} \cong$ $7 \times 10^{-7}$, respectively, where we use our value of $M_{G U T}$ given above in the latter evaluation for definiteness. We ignore such small effects here.

We want however to stress again that the model Planck scale cosmology of Bonanno and Reuter which we use is just that, a model. More work needs to be done to remove from it the type of uncertainties which we just elaborated in our estimate of $\Lambda$. We thank Profs. L. Alvarez-Gaume and W. Hollik for the support and kind hospitality of the CERN TH Division and the WernerHeisenberg-Institut, MPI, Munich, respectively, where a part of this work was done.

\section{References}

[1] S. Weinberg, in General Relativity, an Einstein Centenary Survey, eds. S. W. Hawking and W. Israel, (Cambridge Univ. Press, Cambridge, 1979).

[2] M. Reuter, Phys. Rev. D57 (1998) 971, and references therein.

[3] O. Lauscher and M. Reuter, Phys. Rev. D66 (2002) 025026, and references therein.

[4] E. Manrique, M. Reuter and F. Saueressig, Ann. Phys. 326 (2011) 44 and references therein.

[5] A. Bonanno and M. Reuter, Phys. Rev. D62 (2000) 043008, and references therein.

[6] D. F. Litim, Phys. Rev. Lett.92(2004) 201301; Phys. Rev. D64 (2001) 105007; P. Fischer and D.F. Litim, Phys. Lett. B638 (2006) 497 and references therein.

[7] D. Don and R. Percacci, Class. Quant. Grav. 15 (1998) 3449; R. Percacci and D. Perini, Phys. Rev. D67(2003) 081503; ibid.68 (2003) 044018; R. Percacci, ibid.73(2006) 041501; A. Codello, R. Percacci and C. Rahmede, Int.J. Mod. Phys. A23(2008) 143, and references therein.

[8] K. G. Wilson, Phys. Rev. B4 (1971) 3174, 3184; K. G. Wilson, J.Kogut, Phys. Rep. 12 (1974) 75; F. Wegner, A. Houghton, Phys. Rev. A8(1973) 401; S. Weinberg, "Critical Phenomena for Field Theorists", Erice Subnucl. Phys. (1976) 1; J. Polchinski, Nucl. Phys. B231 (1984) 269.

[9] B.F.L. Ward, Open Nucl.Part.Phys.Jour. 2(2009) 1; arXiv:1008.1046; and references therein.

[10] B.F.L. Ward, Mod. Phys. Lett. A17 (2002) 237. 
[11] B.F.L. Ward, Mod. Phys. Lett. A19 (2004) 143.

[12] B.F.L. Ward, Mod. Phys. Lett. A23 (2008) 3299.

[13] D. R. Yennie, S. C. Frautschi, and H. Suura, Ann. Phys. 13 (1961) 379; see also K. T. Mahanthappa, Phys. Rev. 126 (1962) 329, for a related analysis.

[14] J. Ambjorn et al., Phys. Lett. B690 (2010) 420, and references therein.

[15] P. Horava, Phys. Rev. D 79 (2009) 084008.

[16] A. Bonanno and M. Reuter, Phys. Rev. D65 (2002) 043508.

[17] A. Bonanno and M. Reuter, Jour. Phys. Conf. Ser. 140 (2008) 012008, and references therein.

[18] I. L. Shapiro and J. Sola, Phys. Lett. B475 (2000) 236.

[19] See for example A. H. Guth and D.I. Kaiser, Science 307 (2005) 884; A. H. Guth, Phys. Rev. D23 (1981) 347, and references therein.

[20] See for example A. Linde, Lect. Notes. Phys. 738 (2008) 1, and references therein.

[21] A.G. Riess et al., Astron. Jour. 116 (1998) 1009; S. Perlmutter et al., Astrophys. J. 517 (1999) 565.

[22] C. Amsler et al., Phys. Lett. B667 (2008) 1.

[23] B.F.L. Ward, to appear.

[24] S. Weinberg, The Quantum Theory of Fields, v.1,(Cambridge University Press, Cambridge, 1995).

[25] R.P. Feynman, Acta Phys. Pol.24 (1963) 697-722.

[26] R.P. Feynman, Lectures on Gravitation, eds. F.B. Moringo and W.G. Wagner WG, (Caltech, Pasadena, 1971).

[27] L.D. Faddeev and V.N. Popov, "Perturbation theory for gauge invariant fields", preprint ITF-67-036, NAL-THY-57 (translated from Russian by D. Gordon and B.W. Lee). Available from http://lss.fnal.gov/archive/test-preprint/fermilab-pub-72-057-t.shtml.

[28] Faddeev LD and Popov VN. Feynman diagrams for the Yang-Mills field. Phys Lett B 1967; 25: 29-30.

[29] Ya. B. Zeldovich, Sov. Phys. Uspekhi 11 (1968) 381.

[30] V. Branchina and D. Zappala, G. R. Gravit. 42 (2010) 141; arXiv:1005.3657, and references therein.

[31] J. Sola, J. Phys. A41 (2008) 164066.

[32] P.S. Bhupal Dev and R.N. Mohapatra, Phys. Rev. D82 (2010) 035014, and references therein.

[33] S.L. Glashow, Nucl. Phys. 22 (1961) 579; S. Weinberg, Phys. Rev. Lett. 19 (1967) 1264; A. Salam, in Elementary Particle Theory, ed. N. Svartholm (Almqvist and Wiksells, Stockholm, 1968), p. 367;

G. 't Hooft and M. Veltman, Nucl. Phys. B44,189 (1972) and 50, 318 (1972); G. 't Hooft, ibid. 35, 167 (1971); M. Veltman, ibid. 7, 637 (1968).

[34] D. J. Gross and F. Wilczek, Phys. Rev. Lett. 30 (1973) 1343; H. David Politzer, ibid.30 (1973) 1346; see also, for example, F. Wilczek, in Proc. 16th International Symposium on Lepton and Photon Interactions, Ithaca, 1993, eds. P. Drell and D.L. Rubin (AIP, NY, 1994) p. 593.

[35] See for example H.E. Haber and G.L. Kane, Phys. Rep.117 (1985) 75, and references therein.

[36] See for example S. Lowette, in Proc. Rencontres de Moriond EW 2012, in press. 